\title{
On the Relativistic Foundations of Pseudospin Symmetry in Nuclei
}

\author{
J.N. Ginocchio ${ }^{1, *}$ and A. Leviatan ${ }^{2,1, \dagger}$ \\ 1 Theoretical Division, Los Alamos National Laboratory, Los Alamos, New Mexico 87545, USA \\ ${ }^{2}$ Racah Institute of Physics, The Hebrew University, Jerusalem 91904, Israel
}

\begin{abstract}
We show that the generators of pseudospin symmetry are the non - relativistic limit of the generators of an SU(2) symmetry which leaves invariant the Dirac Hamiltonian with scalar and vector potentials equal in magnitude but opposite in sign, $V_{V}=-V_{S}$. Furthermore, within this framework, we demonstrate that this symmetry may be approximately conserved for realistic scalar and vector potentials.
\end{abstract}

PACS: 24.10.Jv, 21.60.Cs, 24.80.+y, 21.10.-k

Keywords: Relativistic mean field theory; Symmetry; Dirac Hamiltonian; Pseudospin

*E-mail address: gino@t5.lanl.gov

†E-mail address: ami@vms.huji.ac.il 
Pseudospin doublets were introduced almost thirty years ago into nuclear physics to accommodate an observed near degeneracy of certain normal - parity shell-model orbitals with non - relativistic quantum numbers $\left(n_{r}, \ell, j=\ell+1 / 2\right)$ and $\left(n_{r}-1, \ell+2, j=\ell+3 / 2\right)$ where $n_{r}, \ell$, and $j$ are the single-nucleon radial, orbital, and total angular momentum quantum numbers, respectively [1.2]. The doublet structure is expressed in terms of a "pseudo" orbital angular momentum $\tilde{\ell}=\ell+1$ and "pseudo" spin, $\tilde{s}=1 / 2$. For example, $\left(n_{r} s_{1 / 2},\left(n_{r}-1\right) d_{3 / 2}\right)$ will have $\tilde{\ell}=1,\left(n_{r} p_{3 / 2},\left(n_{r}-1\right) f_{5 / 2}\right)$ will have $\tilde{\ell}=2$, etc. These doublets are almost degenerate with respect to pseudospin, since $j=\tilde{\ell} \pm \tilde{s}$ for the two states in the doublet. This symmetry has been used to explain features of deformed nuclei [3], including superdeformation [4] and identical bands [5:6], and to establish an effective shell - model coupling scheme [7]. Therefore there is an interest in understanding the origin of this "symmetry" [8 [0]. The work of [9] has shown that a microscopic normal $\rightarrow$ pseudo transformation leads to an effective reduction of the spin-orbit splitting in the single-particle energy spectra derived from realistic (one boson exchange) internucleon potentials, in line with relativistic mean-field estimates [8]. The work of [10] has shown that quasi-degenerate pseudospin doublets in nuclei arise from the near equality in the magnitudes of an attractive scalar, $V_{S}$, and a repulsive vector, $V_{V}$, relativistic mean fields, $V_{S} \sim-V_{V}$, in which the nucleons move. A near equality in the magnitude of mean fields seems to be a universal feature of relativistic theories ranging from relativistic field theories with interacting nucleons and mesons [11], to nucleons interacting via Skyrme-type interactions [12,13], to QCD sum rules [14]. Recently realistic relativistic mean fields were shown to exhibit this approximate pseudospin symmetry in both the energy spectra and wave functions [15]. In this paper we identify the algebra responsible for pseudospin symmetry as a non - relativistic limit of an $\mathrm{SU}(2)$ symmetry of a Dirac Hamiltonian with $V_{S}=-V_{V}$.

The Dirac Hamiltonian, $\mathrm{H}$, with an external scalar, $V_{S}$, and vector, $V_{V}$, potentials is given by:

$$
H=\boldsymbol{\alpha} \cdot \boldsymbol{p}+\beta\left(m+V_{S}\right)+V_{V},
$$


where we have set $\hbar=c=1$ and $\boldsymbol{\alpha}, \beta$ are the usual Dirac matrices [16]. The Dirac Hamiltonian is invariant under an $\mathrm{SU}(2)$ algebra for two limits: $V_{S}=V_{V}$ and $V_{S}=-V_{V}$ [17 19, $\left(V_{S}, V_{V}\right.$ are spin scalars). The former limit has application to the spectrum of mesons for which the spin - orbit splitting is small. We shall show that the latter limit has relevance to understanding the origin of pseudospin symmetry in nuclei.

The generators for the SU(2) algebra, $\hat{S}_{i}$, which commute with the Dirac Hamiltonian, $\left[H, \hat{S}_{i}\right]=0$, for the case when $V_{S}=-V_{V}$ are given by [18]

$$
\hat{S}_{i}=\frac{\boldsymbol{\alpha} \cdot \boldsymbol{p} \hat{s}_{i} \boldsymbol{\alpha} \cdot \boldsymbol{p}}{p^{2}} \frac{(1+\beta)}{2}+\hat{s}_{i} \frac{(1-\beta)}{2},
$$

where $\hat{s}_{i}=\sigma_{i} / 2$ are the usual spin generators and $\sigma_{i}$ the Pauli matrices. This reduces to

$$
\hat{S}_{i}=\left(\begin{array}{cc}
\hat{\tilde{s}}_{i} & 0 \\
0 & \hat{s}_{i}
\end{array}\right)
$$

where

$$
\hat{\tilde{s}}_{i}=U_{p} \hat{s}_{i} U_{p}=\frac{2 \boldsymbol{s} \cdot \boldsymbol{p}}{p^{2}} p_{i}-\hat{s}_{i}
$$

In (4) $U_{p}=\frac{\boldsymbol{\sigma} \cdot \boldsymbol{p}}{p}$ is the momentum-helicity unitary operator introduced in [9] that accomplishes the transformation from the normal shell model space to the pseudo shell model space while preserving rotational, parity, time - reversal, and translational invariance. What is significant is that the same unitary transformation appears in the non - relativistic limit of the generators of a symmetry possessed by the Dirac Hamiltonian for $V_{S}=-V_{V}$. In this case, the eigenfunctions of the Dirac Hamiltonian, $H \Psi=\mathcal{E} \Psi$ are also doublets $(S=1 / 2$, $\tilde{\mu}= \pm 1 / 2)$ with respect to the $\mathrm{SU}(2)$ generators $\hat{S}_{i}$ of Eq. (3)

$$
\begin{aligned}
\hat{S}_{z} \Psi_{\tilde{\mu}} & =\tilde{\mu} \Psi_{\tilde{\mu}}, \\
\hat{S}_{ \pm} \Psi_{\tilde{\mu}} & =\sqrt{(1 / 2 \mp \tilde{\mu})(3 / 2 \pm \tilde{\mu})} \Psi_{\tilde{\mu} \pm 1}, \quad\left(V_{S}=-V_{V}\right)
\end{aligned}
$$

where $\hat{S}_{ \pm}=\hat{S}_{x} \pm i \hat{S}_{y}$.

In general, the eigenfunctions of the Dirac Hamiltonian, have an upper $\left(\Psi_{+}\right)$and lower $\left(\Psi_{-}\right)$components, $\Psi_{ \pm}=\frac{(1 \pm \beta)}{2} \Psi$. In nuclear spectroscopy, the lower component, which is 
small [15], is usually ignored. The upper component satisfies the second order differential equation,

$$
H_{+} \Psi_{+}=(\mathcal{E}-m) \Psi_{+}
$$

where

$$
H_{+}=H_{+}^{p s}+V_{V}+V_{S}
$$

and the Hamiltonian $H_{+}^{p s}$ is given by

$$
H_{+}^{p s}=\boldsymbol{\sigma} \cdot \boldsymbol{p} \frac{1}{\mathcal{E}+m+V_{S}-V_{V}} \boldsymbol{\sigma} \cdot \boldsymbol{p}
$$

Using the identity $\boldsymbol{\sigma} \cdot \boldsymbol{p} \boldsymbol{\sigma} \cdot \boldsymbol{p}=p^{2}$, it is easy to show that

$$
\left[\hat{\tilde{s}}_{i}, H_{+}^{p s}\right]=0
$$

hence $H_{+}^{p s}$ conserves pseudospin. As seen from Eq. (đ), the $\hat{\tilde{s}}_{i}$ are obtained by a unitary transformation from the ordinary-spin operators $\hat{s}_{i}$, hence generate an $\mathrm{SU}(2)$ algebra

$$
\left[\hat{\tilde{s}}_{i}, \hat{\tilde{s}}_{j}\right]=i \epsilon_{i j k} \hat{\tilde{s}}_{k}
$$

This means that, for $V_{S}=-V_{V}$, the non-relativistic single - nucleon wave functions (Dirac upper components) can then be labeled by pseudospin, $\tilde{s}=1 / 2$, its projection, $\tilde{\mu}$, $\tilde{\mu}=$ $\pm 1 / 2, \Psi_{+, \tilde{\mu}}$, and that these doublets are degenerate. The pseudospin generators connect wave functions of the doublets in the usual way,

$$
\begin{aligned}
& \hat{\tilde{s}}_{z} \Psi_{+, \tilde{\mu}}=\tilde{\mu} \Psi_{+, \tilde{\mu}}, \\
& \hat{\tilde{s}}_{ \pm} \Psi_{+, \tilde{\mu}}=\sqrt{(1 / 2 \mp \tilde{\mu})(3 / 2 \pm \tilde{\mu})} \Psi_{+, \tilde{\mu} \pm 1}, \quad\left(V_{S}=-V_{V}\right)
\end{aligned}
$$

where $\hat{\tilde{s}}_{ \pm}=\hat{\tilde{s}}_{x} \pm i \hat{\tilde{s}}_{y}$.

However, in the exact pseudospin limit, there are no bound Dirac valence states for realistic mean fields [10] and therefore nuclei would not exist if pseudospin symmetry were exact. Nevertheless, it is possible to have small pseudospin symmetry breaking and have 
the requisite number of bound Dirac valence states [10,15] for nuclei to exist. Furthermore, we claim that the relationship between the wave functions of the doublets given in (11) will still be approximately valid. In order to prove this we see from (5), that, in the pseudospin symmetry limit, the lower components are ordinary spin doublets with spin, $s=1 / 2$, and projection $\tilde{\mu}, \tilde{\mu}= \pm 1 / 2$ :

$$
\begin{aligned}
\hat{s}_{z} \Psi_{-, \tilde{\mu}} & =\tilde{\mu} \Psi_{-, \tilde{\mu}} \\
\hat{s}_{ \pm} \Psi_{-, \tilde{\mu}} & =\sqrt{(1 / 2 \mp \tilde{\mu})(3 / 2 \pm \tilde{\mu})} \Psi_{-, \tilde{\mu} \pm 1}, \quad\left(V_{S}=-V_{V}\right) .
\end{aligned}
$$

These spin doublets are degenerate in energy and the wave functions $\Psi_{-, \tilde{\mu}}, \tilde{\mu}= \pm 1 / 2$, have the same dependence on the spatial coordinates in the pseudospin limit. However, in [15, it was shown that, even for realistic mean field Dirac Hamiltonians, the lower components of doublets have almost identical spatial wave functions, particularly for the doublets close to the Fermi sea. Even though [15] considered only spherically symmetric scalar and vector potentials, we assume this to be true in general and therefore label the states with spin projection $\tilde{\mu}$ referring to the dominant spin projection in the wave function. We now show that the pseudospin relations between the wave functions given in (11) for the upper components are approximately valid even when there is symmetry - breaking.

From the Dirac equation, $H \Psi=\mathcal{E} \Psi$, we derive,

$$
\Psi_{+, \tilde{\mu}}=\frac{1}{\left(\mathcal{E}-m-V_{S}-V_{V}\right)} \boldsymbol{\sigma} \cdot \boldsymbol{p} \Psi_{-, \tilde{\mu}} .
$$

Assuming that the lower component is normalized to unity, the normalization of the upper component is then $\left\langle\Psi_{+, \tilde{\mu}} \mid \Psi_{+, \tilde{\mu}}\right\rangle=\mathcal{N}^{-2}=\left\langle\Psi_{-, \tilde{\mu}}\left|\boldsymbol{\sigma} \cdot \boldsymbol{p}\left(\frac{1}{\mathcal{E}-m-V_{S}-V_{V}}\right)^{2} \boldsymbol{\sigma} \cdot \boldsymbol{p}\right| \Psi_{-, \tilde{\mu}}\right\rangle$. For the realistic Dirac equation with $V_{S} \neq-V_{V}$, the lower component satisfies the second order differential equation,

$$
\left[\boldsymbol{\sigma} \cdot \boldsymbol{p} \frac{1}{\left(\mathcal{E}-m-V_{S}-V_{V}\right)} \boldsymbol{\sigma} \cdot \boldsymbol{p}-m+V_{V}-V_{S}\right]\left|\Psi_{-, \tilde{\mu}}\right\rangle=\mathcal{E}\left|\Psi_{-, \tilde{\mu}}\right\rangle .
$$

Using (14), we find that

$$
\mathcal{N}^{-2}=\left\langle\Psi_{-, \tilde{\mu}}\left|\left(\mathcal{E}-V_{V}+V_{S}+m\right) \frac{1}{p^{2}}\left(\mathcal{E}-V_{V}+V_{S}+m\right)\right| \Psi_{-, \tilde{\mu}}\right\rangle .
$$


The normalized upper component will then be $\Phi_{+, \tilde{\mu}}=\mathcal{N} \Psi_{+, \tilde{\mu}}$. Using similar manipulations, we then can derive the matrix elements of the pseudospin operators to be,

$$
\begin{aligned}
\left\langle\Phi_{+, \tilde{\mu}^{\prime}}\left|\tilde{\hat{s}}_{i}\right| \Phi_{+, \tilde{\mu}}\right\rangle & =\mathcal{N}^{2}\left\langle\Psi_{-, \tilde{\mu}^{\prime}}\left|\left(\mathcal{E}-V_{V}+V_{S}+m\right) \frac{\hat{s}_{i}}{p^{2}}\left(\mathcal{E}-V_{V}+V_{S}+m\right)\right| \Psi_{-, \tilde{\mu}}\right\rangle \\
& \approx\left\langle\Psi_{-, \tilde{\mu}^{\prime}}\left|\hat{s}_{i}\right| \Psi_{-, \tilde{\mu}}\right\rangle
\end{aligned}
$$

where the last step follows from the fact that the lower component has the spin and spatial parts approximately separated [15] and from (15). Therefore, the near identity of the spatial part of the lower component wave function for the pseudospin doublet insures that the pseudospin doublets are approximately connected by the pseudospin generators even for realistic situations in which pseudospin is broken:

$$
\begin{aligned}
\left\langle\Phi_{+, \tilde{\mu}^{\prime}}\left|\hat{\tilde{s}}_{z}\right| \Phi_{+, \tilde{\mu}}\right\rangle & \approx \tilde{\mu} \delta_{\tilde{\mu}^{\prime}, \tilde{\mu}}, \\
\left\langle\Phi_{+, \tilde{\mu}^{\prime}}\left|\hat{\tilde{s}}_{ \pm}\right| \Phi_{+, \tilde{\mu}}\right\rangle & \approx \sqrt{(1 / 2 \mp \tilde{\mu})(3 / 2 \pm \tilde{\mu})} \delta_{\tilde{\mu}^{\prime}, \tilde{\mu} \pm 1}, \quad\left(V_{S} \approx-V_{V}\right) .
\end{aligned}
$$

In the case in which the potentials are spherically symmetric and satisfy $V_{S}=-V_{V}$, the Dirac Hamiltonian has an additional invariant SU(2) algebra; namely,

$$
\hat{L}_{i}=\left(\begin{array}{cc}
\hat{\tilde{\ell}}_{i} & 0 \\
0 & \hat{\ell}_{i}
\end{array}\right),
$$

where $\hat{\tilde{\ell}}_{i}=U_{p} \hat{\ell}_{i} U_{p}$ is the pseudo-orbital angular momentum operator, $\hat{\ell}_{i}$ is the orbital angular momentum operator, while $\hat{j}_{i}=\hat{\tilde{\ell}}_{i}+\hat{\tilde{s}}_{i}=U_{p}\left(\hat{\ell}_{i}+\hat{s}_{i}\right) U_{p}=\hat{\ell}_{i}+\hat{s}_{i}$. In this limit, the Dirac wave functions are eigenfunctions of the Casimir operator of this algebra, $\hat{\boldsymbol{L}} \cdot \hat{\boldsymbol{L}}\left|\Phi_{\tilde{\ell}, j, m_{j}}\right\rangle=$ $\tilde{\ell}(\tilde{\ell}+1)\left|\Phi_{\tilde{\ell}, j, m_{j}}\right\rangle$, where we have used a coupled basis, $\vec{j}=\overrightarrow{\tilde{\ell}}+\overrightarrow{\tilde{s}}$, where $j$ is the eigenvalue of the total angular momentum operator $\hat{J}_{i}=\hat{L}_{i}+\hat{S}_{i}, \hat{\boldsymbol{J}} \cdot \hat{\boldsymbol{J}}\left|\Phi_{\tilde{\ell}_{j, j} m_{j}}\right\rangle=j(j+1)\left|\Phi_{\tilde{\ell}, j, m_{j}}\right\rangle$, and $m_{j}$ is the eigenvalue of $\hat{J}_{z}$. Thus pseudo-orbital angular momentum as well as pseudospin are conserved in the spherical limit and $V_{S}=-V_{V}$. (We note in passing that the operator $\hat{K}=-\beta(\boldsymbol{\sigma} \cdot \hat{\boldsymbol{\ell}}+1)$ which is conserved for arbitrary spherically symmetric $V_{S}$ and $V_{V}$ potentials 16 is given by $\hat{K}=2 \hat{\boldsymbol{L}} \cdot \hat{\boldsymbol{S}}+1$ ). From (18), we see that the lower component wave function will have spherical harmonic of $\operatorname{rank} \tilde{\ell}$ coupled to spin to give total angular momentum $j$. Since $\boldsymbol{\sigma} \cdot \boldsymbol{p}$ conserves the total angular momentum but $\boldsymbol{p}$ changes the orbital 
angular momentum by one unit because of parity conservation, Eq. (13) tells us that the the upper component also has total angular momentum $j$, but orbital angular momentum $\ell=\tilde{\ell} \pm 1$. If $j=\tilde{\ell}+1 / 2$, then it follows that $\ell=\tilde{\ell}+1$, whereas if $j=\tilde{\ell}-1 / 2$, then $\ell=\tilde{\ell}-1$. This agrees with the pseudospin doublets originally observed [1,2] and discussed at the beginning of this paper. However, the results here are very general and apply to non - spherical nuclei as well [3,20]. For example, for axially symmetric deformed nuclei, there is a U(1) generator corresponding to the pseudo-orbital angular momentum projection along the symmetry axis which is conserved in addition to the pseudospin for $V_{S}=-V_{V}$,

$$
\hat{\lambda}=\left(\begin{array}{cc}
\hat{\tilde{\Lambda}} & 0 \\
0 & \hat{\Lambda}
\end{array}\right),
$$

where $\hat{\tilde{\Lambda}}=U_{p} \hat{\Lambda} U_{p}$. In this case the Dirac wave functions are eigenfunctions of $\hat{\lambda}, \hat{\lambda}\left|\Phi_{\tilde{\Lambda}, \Omega}\right\rangle=$ $\tilde{\Lambda}\left|\Phi_{\tilde{\Lambda}, \Omega}\right\rangle$, where $\Omega$ is the total angular momentum projection, $\Omega=\tilde{\Lambda}+\tilde{\mu}$, which has the same value for the upper and lower components since $\boldsymbol{\sigma} \cdot \boldsymbol{p}$ conserves the total angular momentum projection. Thus $\Omega=\tilde{\Lambda} \pm 1 / 2$, corresponding exactly to the quantum numbers of the pseudospin doublets for axially deformed nuclei discussed in [3]. Again we expect these multiplet relationships to be approximately valid for the realistic situation, $V_{S} \approx-V_{V}$, following our previous discussion.

The unitary transformation, $U_{p}$ (田), has been used to transform the non-relativistic spherical shell model space to the pseudo shell model space [9]. Basically, as seen from Eq. (13) and subsequent discussion, in the limit of $V_{S}=-V_{V}$, this transformation transforms the normalized Dirac upper component wave functions into the normalized Dirac lower components.

The Hamiltonian for the upper components in the realistic case which is given in (7), leads to a Schrödinger equation,

$$
\begin{gathered}
{\left[\frac{1}{\left(2 m+V_{S}-V_{V}\right)} p^{2}+\frac{1}{\left(2 m+V_{S}-V_{V}\right)^{2}}\left[\boldsymbol{\sigma} \cdot \boldsymbol{p}, V_{V}-V_{S}\right] \boldsymbol{\sigma} \cdot \boldsymbol{p}+V_{V}+V_{S}\right]\left|\Phi_{+, \tilde{\mu}}\right\rangle} \\
=(\mathcal{E}-m)\left|\Phi_{+, \tilde{\mu}}\right\rangle
\end{gathered}
$$

where we have made the approximation that $\left(2 m+V_{S}-V_{V}\right)>>m-\mathcal{E}$, the binding 
energy. This Schrödinger equation is not of the form that follows from the usual shell model single - nucleon Hamiltonian because it has a spatial-dependent mass term and, since $\left(V_{V}-V_{S}\right) / 2 m \approx 0.41[12$ in the nuclear interior, the spatial dependence cannot be neglected. Furthermore, the second term in (20), which produces a spin - orbit term for spherically symmetric potentials, produces a momentum-dependent spin term as well. Although the helicity transformation of non-relativistic momentum-independent Hamiltonians [9] and shell-model wave functions [21] induces momentum dependence, the above analysis demonstrates that the non-relativistic wave function (Dirac upper component) has momentum dependence before the helicity transformation since it is a solution of a momentum-dependent Hamiltonian with a spatially dependent effective mass.

One can ask if there are tests of the pseudospin doublet wave functions. One ingenious attempt was applied to polarized medium energy proton scattering for which the scalar optical potential is approximately equal and opposite in sign to the vector optical potential [22]. Pseudospin predictions of the spin polarization and spin rotation functions were shown to be badly broken experimentally. The reason for this is as follows. In the strict symmetry limit the scattering functions for the partial waves depends only on $\tilde{\ell}$, not on the pseudospin and hence are equal for pseudospin doublets. When the symmetry is broken the difference in the scattering functions for doublets is proportional to $2 \tilde{\ell}+1$, just like the energy splitting for pseudospin doublets for bound orbitals [10,15]. For medium energy proton scattering large orbital angular momentum, and hence, large pseudo - orbital angular momentum, dominate the scattering whereas in heavy nuclei the occupied orbitals have relatively small pseudo orbital angular momenta (for example, the largest for an occupied bound orbital in ${ }^{208} \mathrm{~Pb}$ is $\tilde{\ell}=4$ ). For this reason tests of the bound state wave functions may be more fruitful.

In summary, we have shown that a relativistic symmetry of the Dirac Hamiltonian with $V_{V}=-V_{S}$ reduces to the pseudospin symmetry in the non - relativistic limit. We have suggested that the pseudospin doublet wave functions of Dirac's upper components may still possess this symmetry even in the realistic $V_{V} \approx-V_{S}$ situation. These results are expected to apply to non - spherical as well as spherical nuclei, in line with the validity 
of the pseudospin concept shown for the oscillator shell model with arbitrary quadrupole deformations [20,21]. We also conclude that the conventional non - relativistic shell model single-nucleon Hamiltonian could be made to be consistent with the relativistic mean field, and, as a consequence, pseudospin symmetry, by including a spatial - dependent mass and a momentum-dependent spin term in the mean field.

We thank A. S. Goldhaber for discussions and for informing us about references 17 19,22. This research was supported in part by the United States Department of Energy and in part by a grant from the Israel Science Foundation. 


\section{REFERENCES}

[1] K.T. Hecht and A. Adler, Nucl. Phys. A137 (1969) 129.

[2] A. Arima, M. Harvey and K. Shimizu, Phys. Lett. B30 (1969) 517.

[3] A. Bohr, I. Hamamoto and B. R. Mottelson, Phys. Scr. 26 (1982) 267.

[4] J. Dudek, W. Nazarewicz, Z. Szymanski and G. A. Leander, Phys. Rev. Lett. 59 (1987) 1405.

[5] W. Nazarewicz, P. J. Twin, P. Fallon and J.D. Garrett, Phys. Rev. Lett. 64 (1990) 1654.

[6] B. Mottelson, Nucl. Phys. A522 (1991) 1.

[7] D. Troltenier, C. Bahri and J. P. Draayer, Nucl. Phys. A586 (1995) 53.

[8] C. Bahri, J.P. Draayer and S.A. Moszkowski, Phys. Rev. Lett. 68 (1992) 2133.

[9] A. L. Blokhin, C. Bahri and J. P. Draayer, Phys. Rev. Lett. 74 (1995) 4149.

[10] J. N. Ginocchio, Phys. Rev. Lett. 78 (1997) 436.

[11] B. D. Serot and J. D. Walecka, The Relativistic Nuclear Many - Body Problem in Advances in Nuclear Physics, edited by J. W. Negele and E. Vogt, Vol. 16 (Plenum, New York, 1986).

[12] B. A. Nikolaus, T. Hoch and D. G. Madland, Phys. Rev. C46 (1992) 1757.

[13] D. G. Madland, in Proceedings of the International Conference on Nuclear Data for Science and Technology, Trieste, Italy, May 19-24, 1997 (in press). [See Table 4, Calculation (b), and discussion.]

[14] T. D. Cohen, R. J. Furnstahl and D. K. Griegel, Phys. Rev. Lett. 67 (1991) 961.

[15] J. N. Ginocchio and D. A. Madland, submitted to Phys. Rev. C (1997). 
[16] W. Greiner, B. Müller and J. Rafelski, Quantum Electrodynamics of Strong Fields (Springer-Verlag, New York, 1985).

[17] G. B. Smith and L. J. Tassie, Ann. Phys. 65 (1971) 352.

[18] J. S. Bell and H. Ruegg, Nucl. Phys. B98 (1975) 151.

[19] G. Eyre and H. Osborn, Nucl. Phys. B116 (1976) 281.

[20] T. Beuschel, A. L. Blokhin and J. P. Draayer, Nucl. Phys. A619 (1997) 119.

[21] A.L. Blokhin, T. Beuschel, J.P. Draayer and C. Bahri, Nucl. Phys. A612 (1997) 163.

[22] J. B. Bowlin, A. S. Goldhaber and C. Wilkin, Z. Phys. A331 (1988) 83. 